Published in final edited form as:

Respirology. 2013 April ; 18(3): 573-583. doi:10.1111/resp.12048.

\title{
Year in review 2012: Lung cancer, respiratory infections, tuberculosis, pleural diseases, bronchoscopic intervention and imaging
}

\author{
José M. Porcel ${ }^{1}$, Chi Chiu Leung ${ }^{2}$, Marcos I. Restrepo ${ }^{3}$, Kazuhisa Takahashi ${ }^{4}$, and Pyng \\ Lee $^{5}$ \\ ${ }^{1}$ Pleural Diseases Unit, Department of Internal Medicine, Arnau de Vilanova University Hospital, \\ Biomedical Research Institute of Lleida, Lleida, Spain \\ ${ }^{2}$ Tuberculosis and Chest Service, Department of Health, Hong Kong, China \\ 3University of Texas Health Science Center at San Antonio, San Antonio, TX; South Texas \\ Veterans Health Care System Audie L. Murphy Division, and Veterans Evidence Based Research \\ Dissemination and Implementation Center (VERDICT), San Antonio Texas, USA \\ ${ }^{4}$ Department of Respiratory Medicine, Juntendo University Graduate School of Medicine, Hongo, \\ Bunkyo-ku, Tokyo, Japan \\ ${ }^{5}$ Department of Medicine, National University Hospital, Singapore
}

\begin{abstract}
Keywords
lung cancer; respiratory infections; tuberculosis; pleural diseases; bronchoscopic intervention and imaging
\end{abstract}

\section{INTRODUCTION}

This Year in Review series addresses the most relevant articles published in Respirology and other respiratory medicine journals during 2012 concerning five specific areas that we consider to be of importance to practicing pulmonologists, namely lung cancer, respiratory infections, tuberculosis (TB), pleural diseases, and interventional pulmonology and imaging. Some important findings that will be commented on more in depth are that: 1) screening for lung cancer using low-dose computed tomography (CT) in high risk populations is promising, although not firmly established, 2) an enhanced CURB (which stands for confusion, urea, respiratory rate, blood pressure) score as well as the Japanese A-DROP (age, dehydration, respiratory failure, orientation disturbance, and pressure) prognostic scale are as accurate as the pneumonia severity index (PSI) scoring system to predict mortality in patients with community-acquired pneumonia (CAP), 3) randomized trials are urgently needed to optimize multidrug-resistant (MDR)-TB treatment, 4) the use of video-assisted thoracoscopic surgery (VATS) to quantify pleural tumor burden and, if feasible, perform an intrathoracic cytoreduction in patients with malignant effusions secondary to ovarian cancer (OC) may have a significant impact on further patient management plans, and 5) respiratory

Correspondence: José M. Porcel, Department of Internal Medicine, Arnau de Vilanova University Hospital, Avda Alcalde Rovira Roure 80, 25198 Lleida, Spain, jporcelp@yahoo.es.

Conflict of interest statement:

The authors have no conflicts of interest to declare. 
endoscopy and its different diagnostic and therapeutic modalities, is a safe procedure with overall complication rates less than $1 \%$.

\section{LUNG CANCER}

\section{Kazuhisa Takahashi}

Lung cancer is the leading cause of cancer-related death worldwide. ${ }^{1}$ This section will review recent papers regarding the prevention of lung cancer, molecular mechanisms, new diagnostic techniques, translational research and treatments for lung cancer.

Prevention-Based on a review by Lim W et al., approximately $40 \%$ of the world's population depends on biomass fuels (wood, charcoal, dung, crop residue) for cooking and heating. ${ }^{2}$ This burden is particularly high among Asian countries. The authors summarized the biological and epidemiological evidence of biomass fuel emissions on lung cancer development. Among several epidemiologic reports, Lissowska's case-controlled study ${ }^{3}$ and a pool of seven case-controlled studies ${ }^{4}$ published for the International Lung Cancer Consortium convincingly revealed an increased risk of lung cancer due to wood smoke exposure with an odds ratio of 1.21-1.31.

Recently, a number of genome-wide association studies identified several chromosomal regions that contain genes associated with the risk of lung cancer in populations of European descent. Bae et al. examined and confirmed the association between single-nucleotide polymorphisms (SNPs) in the 5p15 and 5q25 chromosomal regions and lung cancer risk in a Korean population, findings that have already been identified in Europe ${ }^{5}$. Specifically, re2736100, CLPTM1L rs402710 and rs401681 SNPs in the 5p15 region have been found to be significantly associated with the risk of lung cancer and, therefore, populations harboring these genetic variants should be strongly advised to stop smoking and receive careful follow-up.

Screening and early diagnosis-Screening for lung cancer using chest X-ray has not shown long-term benefits in terms of overall mortality. The increased sensitivity of CT scanning has rekindled the promotion of early detection and has led to several hypothesisgenerating studies. However, until the results of the American National Lung Cancer Screening Trial (NLST) were published, whether screening using CT scanning could reduce lung cancer mortality remained unknown. The findings of the NLST were reported in 2011 and revealed that low-dose CT screening reduces mortality due to lung cancer by $20 \%$ and all-cause mortality by $6.7 \%$ compared with chest radiograph screening. ${ }^{6}$ Spiro et al. reviewed this topic by evaluating the NLST as well as other studies. ${ }^{7}$ In the Italian DNATE study, it was reported that the CT arm contained more patients with low-stage and resectable diseases, although the effects of screening on lung cancer mortality in this study have not yet been reported. ${ }^{8}$ The results of the French DEPISCAN study, which enrolled 1,000 males and females who had smoked more than 15 cigarettes per day for 20 years or had quit smoking within the last 15 years and were currently attending general practices, have not yet been reported. ${ }^{9}$ The authors emphasized that many pitfalls and shortcomings of CT screening remain to be overcome, including overdiagnosis bias, cost-effectiveness, false-positive findings of multiple noncalcified nodules and the willingness of relevant populations to accept CT scanning before its success can be confirmed.

The use of endobronchial ultrasound (EBUS)-guided needle aspiration (TBNA) to determine lymph node (LN) staging in lung cancer patients has become popular. Shmid-Bindert et al. prospectively evaluated the ability of six ultrasound criteria and a sum score model to predict malignancy in mediastinal LN. ${ }^{10}$ The six criteria were as follows: short axis $>1 \mathrm{~cm}$, heterogeneous pattern, round shape, distinct margin, absence of a central hilar structure and 
medium to high blood flow in the LN. If more than two criteria were present, the LN was classified as exhibiting a high risk of malignancy. Two hundred and eighty-one LNs in 145 patients were analyzed. Echogenicity performed the best among all the variables, exhibiting high sensitivity, specificity and positive and negative predictive values $(78 \%, 77 \%, 73 \%$ and $80 \%$, respectively). The probability of malignancy based on the number of positive criteria (the sum score) was approximately $80 \%$ when all criteria were met and less than $10 \%$ when only two criteria or less were met. Accordingly, criteria based on EBUS-TBNA can be helpful for increasing the accuracy of diagnosing LN metastasis.

Recently, two epoch-making update revisions were made in the field of lung cancer: the Tumor, Node, Metastasis (TNM) system for classifying lung cancer, and the new classification of lung adenocarcinoma. Marshall et al. reported that the $7^{\text {th }}$ edition TNM staging system for lung cancer $^{11}$ requires further development because nonanatomic prognostic factors such as age, performance status, histological grade and serum markers are lacking. Moreover, other variables such as the standardized uptake value (SUV) obtained on FDG-PET scanning and molecular profiles such as the EGFR status may need to be prospectively evaluated in the next revision.

Lung adenocarcinomas exhibit a wide spectrum of clinical, molecular and histological features. The European Respiratory Society (ERS) and the American Thoracic Society (ATS) sponsored a new classification study that presented several modifications to the 2004 WHO criteria for the diagnosis of resected adenocarcinoma. ${ }^{12}$ Kadara et al. reviewed recent advances in the molecular pathology of lung adenocarcinoma with an emphasis on genomics and DNA alterations. ${ }^{13}$ Lung adenocarcinomas exhibit unique genomic aberrations compared with lung squamous cell carcinomas. Mutations in the K-ras, EGFR and Her2/

Neu oncogenes occur almost exclusively in adenocarcinomas. An increased gene dosage and protein expression of thyroid transcriptional factor-1/NK2 homeobox 1 (TTF-1/NKX2-1) are prevalent in patients with lung adenocarcinoma. The ALK fusion gene, the cMet amplification, the ROS-1 rearrangement and the RET fusion gene were recently identified as being powerful driver oncogenes in this tumor type. The authors ultimately suggested that comprehensive analyses of early molecular events in the pathogenesis of lung adenocarcinoma will undoubtedly reveal biomarkers that can aid prevention through the use of personalized strategies in the future.

Interestingly, a case of large-cell neuroendocrine carcinoma with the EGFR mutation that may have transformed from an adenocarcinoma was recently reported. ${ }^{14}$ This suggests that not only adenocarcinoma, but also other histological types of lung cancer may need to be evaluated for the EGFR mutation status because the treatment strategy is dependent on the molecular profile.

Translational Research-Chen et al. reviewed biomarkers and transpriptome profiling in patients with lung cancer. ${ }^{15}$ Although accumulated studies have suggested that biomarkers can be used in the clinical setting, none are routinely used in clinical practice, except for EGFR-activating mutations. The authors indicated that large-scale prospective trials are urgently needed to validate previously identified biomarkers. The expression of excision repair cross-complementation group 1 (ERCC1) is known to be a favorable prognostic marker in patients who have undergone surgery for non-small cell lung cancer (NSCLC), but a poor predictive marker for cisplatin response. ${ }^{16}$ Tseden-ish M et al. assessed the prognostic role of ERCC1 expression, SNPs, ERCC1 and class III beta tubulin (TUBB3) in patients with lung cancer who underwent surgery followed by adjuvant chemotherapy. ${ }^{17}$ They revealed that the ERCC1 expression and the AA/CA genotype at C8092A are correlated with good prognoses. 
The epithelial-to-mesenchymal transition (EMT) contributes to the development of various malignant features in cancer cells, including motile, invasive, anti-apoptotic and stem-like phenotypes. ${ }^{18,19}$ According to Sato $\mathrm{M}$ et al. review, EMT may occur during lung cancer development. ${ }^{20}$ Numerous studies have reported that the loss of E-cadherin is quite common and correlates with poor prognoses in patients with lung cancer. ${ }^{21}$ In addition, it has been shown that the expression of molecules involved in EMT correlates with the clinicopathological features of NSCLC. ${ }^{22}$ Targeting the EMT pathway appears to be a very promising therapeutic strategy for treating lung cancer because EMT is involved in most of the important malignant properties of cancer cells.

Giroux Leprieur et al. investigated factors associated with long-term survival (>2 years) among patients with advanced NSCLC and identified new factors, including the administration of maintenance therapy, surgery, a time to first progression of the tumor of $>3$ months and a performance status of $0-1$ at first progression of the tumor. ${ }^{23}$ Therefore, assessing these factors may identify populations of patients with NSCLC that are likely to exhibit prolonged life expectancies.

Mesothelioma-Malignant pleural mesothelioma (MPM) exhibits a poor prognosis. Although treatment with a combination of pemetrexed and cisplatin has been approved, the overall survival of patients with MPM has not been dramatically prolonged. Consequently, new effective molecular targeted drugs are urgently required. One study reported that SU6668, a multiple tyrosine kinase inhibitor, exhibited antitumor effects against human MPM cells and revealed prolonged survival in an orthotopic implantation model of human MPM. ${ }^{24}$ These findings suggest that SU6668 is a useful therapeutic candidate to target human MPM.

\section{RESPIRATORY INFECTIONS}

\section{Marcos I. Restrepo}

During the past year a significant number of manuscripts have been published in Respirology, advancing the science and understanding of the epidemiology, microbiology, diagnosis, treatment and prevention of respiratory infections.

\section{Etiology in respiratory infections: viruses and atypical pathogen identification}

-An invited review by Hsu et al. ${ }^{25}$ assessed the evidence regarding the vulnerability associated with influenza infection in patients with chronic airway diseases such as asthma and chronic obstructive pulmonary disease (COPD). The worsening of their pre-existing chronic airway diseases is associated with more severe symptoms. Recent advances in reverse genetics and innate immunity have revealed several influenza virulence and host factors involved in viral pathogenesis and immune responses. Early innate immunity plays a critical role in limiting viral infection and spread, but the underlying mechanisms leading to enhanced susceptibility to influenza infection and severe symptoms remain unclear.

The identification and clinical suspicion of atypical pathogens causing respiratory infections, particularly pneumonia, is a topic of interest due to the importance of appropriate empirical antimicrobial coverage. Miyashita et al. ${ }^{26}$ showed that it is difficult to distinguish between atypical pneumonia and bacterial pneumonia in elderly patients using the Japanese Respiratory Society (JRS) scoring system. ${ }^{27}$ In addition, Yin et al. ${ }^{28}$ assessed whether the JRS guidelines for CAP were effective for diagnosing atypical pneumonia caused by Mycoplasma pneumoniae. The authors used bacterial culture and real-time polymerase chain reaction (PCR) techniques to detect $M$. pneumoniae, urine antigen tests to detect Streptococcus pneumoniae and Legionella pneumoniae, blood and sputum culture for bacteria, and real-time PCR for eight common respiratory viruses in outpatients with 
pneumonia. An etiological diagnosis was obtained in nearly $50 \%$ of 215 adults with CAP. The JRS scoring system ( $\geq 4$ points) ${ }^{27}$ had a sensitivity of $89 \%$ and specificity of $77 \%$ for identifying $M$. pneumoniae pneumonia. The score includes 6 parameters (1 point each): $<60$ years of age, minor or absent underlying diseases, stubborn cough, adverse findings on chest auscultation, absence of sputum or identifiable etiological agent by rapid diagnostic testing, and peripheral leukocyte count $<10 \times 10^{9} / \mathrm{L}$.

Pneumonia severity assessment-There is significant interest in identifying tools that may assist clinicians in the decision to admit patients with pneumonia to the hospital, inhospital transfers, and predicting clinical outcomes such as mortality and intensive care unit admission. Abisheganaden et al. ${ }^{29}$ determined whether CURB enhanced with a small number of additional variables could predict 30-day mortality with at least the same accuracy as the PSI score. In a retrospective review of hospitalized adults with CAP aged 55 years or older over a 1 year period, the 30 -day mortality was $17.2 \%$ among 1,052 hospital admissions of CAP patients. When age and three co-morbid conditions (metastatic cancer, solid tumors without metastases, and stroke) were added to CURB, the area under the curve (AUC) improved to 0.80 (95\% CI: 0.77-0.83), a predictive accuracy similar to that of PSI. Although interesting, these data require further confirmation before they can be applied in clinical practice. Another study by Kasamatsu et al. ${ }^{30}$ assessed the value of a semiquantitative procalcitonin test and the A-DROP prognostic scale proposed by the JRS for predicting mortality among adults hospitalized with CAP. The AUCs to predict survival were 0.80 for procalcitonin, 0.88 for A-DROP, 0.88 for CURB-65, and 0.89 for PSI. The risk of 30-day mortality was significantly higher in procalcitonin-positive ( $\searrow 0.5 \mathrm{ng} / \mathrm{mL}$ ) than in procalcitonin-negative patients (log-rank test, $\mathrm{P}<0.001)$.

Initial hospital triage, which may have implications in clinical outcomes, is a challenge for clinicians. Brown et al. ${ }^{31}$ observed that admitting a patient with severe CAP to the hospital ward followed by a (delayed) intensive care unit transfer within $72 \mathrm{~h}$ increased 30-day mortality two-fold. These data suggest that intensive monitoring of ward-admitted patients with CAP is warranted.

Antibiotics-Multiple clinical practice guidelines ${ }^{27,32,33}$ recommend appropriate therapies for patients with CAP. However, there might be a gap between the recommended therapies and what clinicians do in practice. For example, one Korean population-based study evaluated antibiotic prescriptions among 3,662 hospitalized adults with CAP (52\% males, $56 \%$ aged $\checkmark 65) .{ }^{34}$ The most frequently prescribed antimicrobial regimens were beta-lactam/ beta-lactamase inhibitors and fluoroquinolones in combination (31\%), and beta-lactam/betalactamase inhibitors plus macrolides (30\%). Single antimicrobial therapies were used in $17 \%$ of the patients, which included cephalosporins (6\%), fluoroquinolones (3.5\%), betalactam/beta-lactamase inhibitors (2\%) and macrolides (2\%). The authors concluded that there was an important divergence from the 2009 Korean guidelines for the treatment of CAP, reinforcing the need for assessing physicians' adherence to the guidelines.

Medication adherence remains a factor that is difficult to measure and has implications in clinical improvement and cure of patients with respiratory infections. Llor et al. ${ }^{35}$ compared the adherence of patients taking a pharmacokinetically enhanced formulation of amoxicillin/ clavulanic acid twice daily with that of those taking the standard formulation thrice daily. The percentage of patients who opened the container a satisfactory number of times per day was significantly higher among those taking the twice-daily regimen on days $3,4,5,6$, and 7. Moreover, the thrice-daily group more frequently forgot to take the afternoon dose. The rate of compliance with amoxicillin/clavulanic acid therapy was very low, but improved significantly with the new formulation taken twice-daily. 
Immunomodulation-The immunomodulatory properties of macrolides and fluoroquinolones may have potential effects on clinical outcomes. Hodge and Reynolds ${ }^{36}$ reported that administration of low-dose azithromycin to subjects with COPD improved macrophage phagocytosis of apoptotic airway epithelial cells (efferocytosis), reduced inflammation and increased expression of macrophage mannose receptors. Phagocytosis of bacteria by both alveolar and monocyte-derived macrophages was also shown to be improved by the antibiotic effect, thereby supporting long-term use of low-dose azithromycin as an attractive adjunct treatment option for COPD. ${ }^{37,38}$ Improved clearance of both apoptotic cells and bacteria in the airway may reduce the release of toxic cell content which perpetuates inflammation and contributes to COPD exacerbations. ${ }^{39}$ In another study, it was demonstrated that there was a downregulation of Th1/Th17/naturally occurring Treg responses after treatment with low-dose clarithromycin in a mouse model of chronic $P$. aeruginosa lung infection. ${ }^{40} \mathrm{In}$ addition, Kawai et al. ${ }^{41}$ presented data on the lower efficacy of macrolides against genetically determined macrolide-resistant $M$. pneumoniae pneumonia in pediatric patients. Finally, Li et al. ${ }^{42}$, investigated, in an animal model, the effects of moxifloxacin on cytokine secretion and signal transduction mechanisms in naive control and allergen-exposed airway smooth muscle cell (ASMC) stimulated with tumor necrosis factoralpha. Moxifloxacin suppressed the secretion of pro-inflammatory cytokines by allergenexposed rat ASMC, partly by inhibiting nuclear factor-kappa B and extracellular-signalregulated kinase activation. They also suggested that dexamethasone may have additional or synergistic effects with moxifloxacin.

Miscellaneous-Smith and Kauffman ${ }^{43}$ reviewed the diagnosis, treatment and epidemiology of pulmonary fungal infections. Advances on this subject have occurred because of increasing knowledge regarding the fungal genome, better understanding of the structures of the fungal cell wall and cell membrane, and the use of molecular epidemiological techniques. This has resulted in more rapid diagnosis and more effective and less toxic antifungal agents.

Hardak et al. ${ }^{44}$ retrospectively described the clinical manifestations, outcomes and factors associated with mortality due to Pneumocystis pneumonia, as diagnosed by PCR, in patients without HIV infection. Overall in-hospital mortality was found to be $17 \%$, much lower than previously reported. Mortality was associated with co-infections, high lactate dehydrogenase levels, female gender, and higher PSI and acute physiology and chronic health evaluation (APACHE) III scores. The authors hypothesized that lower mortality may be due to increased sensitivity of PCR-based assays, as compared with traditional methods, for the diagnosis of pneumocystosis in HIV-negative patients.

Hojo et al. ${ }^{45}$ showed an increased risk of nontuberculous mycobacterial (NTM) infections in asthmatic patients using long-term inhaled corticosteroid therapy. Among 464 asthmatic patients, 14 experienced complications due to NTM infections, of which 8 were caused by Mycobacterium avium-intracellulare complex, 3 by $M$. kansasii, 1 by $M$. terrae and the remaining 2 by unclassifiable scotochromogens. All except one had received inhaled corticosteroid therapy for more than 5 years, and 12 of the 14 patients used inhaled fluticasone propionate daily ( 4 patients at a dose of $400 \mu \mathrm{g} /$ day and 8 patients at a dose $>800$ $\mu \mathrm{g} / \mathrm{day})$. The risk of NTM infection was greater not only in asthmatic patients who received high doses of inhaled corticosteroid therapy, but also in those who were older or had severe airflow limitation. 


\section{TUBERCULOSIS}

\section{Chi Chiu Leung}

Susceptibility to TB-Vitamin D deficiency has been associated with the development of active TB. However, in a South Korean study, ${ }^{46}$ the median serum 25 -hydroxyvitamin D (25-OHD) concentration was no different in TB patients at diagnosis compared with control subjects, thus casting doubt on the contribution of vitamin D deficiency to the overall incidence of TB in a developed economy. On the other hand, a decrease in the median 25OHD concentration was observed with treatment, suggesting an effect of TB on vitamin D metabolism.

Sun et al. found a higher frequency of CD4+CD25+CD127- T-cells (regulatory T cells) in Chinese patients with TB than in normal controls. ${ }^{47}$ Furthermore, in TB patients with diabetes, the frequency of regulatory $\mathrm{T}$ cells was also higher in bronchoalveolar lavage fluid than in peripheral blood, yet similar frequencies of these cells were found in both sites in TB patients without diabetes. In addition, IL-10 expression was significantly higher, and IFN- $\gamma$ expression was significantly lower in bronchoalveolar lavage of TB patients with diabetes than those without. Imbalance between regulatory and effector T-cells at sites of lesions in TB patients with diabetes might potentially weaken local immunity and modulate clinical manifestations.

Dendritic cell-specific intracellular adhesion molecule-3 grabbing nonintegrin (DC-SIGN) encoded by the CD209 gene is a major Mycobacterium tuberculosis receptor on human dendritic cells. In a meta-analysis of 10 studies involving 2,598 TB patients and 2,614 controls, no significant association was found between the CD209-336A/G polymorphism and risk of TB. ${ }^{48}$ Consistent absence of association was observed in the subgroups of African ethnicity and HIV-negative individuals.

Latent TB-Among 101 close contacts of patients with bacteriologically confirmed MDRTB in South Korea, high positive rates were found for tuberculin skin test (TST) using a cutoff of $10 \mathrm{~mm}(47.5 \%)$ and QuantiFERON TB-Gold In-Tube (QFT-GIT) assays (53.5\%). ${ }^{49}$ However, overall agreement between the two tests was poor (kappa, $\kappa=0.33$ ). Lower $\kappa$ measure was observed in subjects with bacillus Calmette-Guerin (BCG) vaccination $(\kappa=0.29)$ than in those without $(\kappa=0.48)$, likely related to the selective effect of BCG on the TST.

In a longitudinal study in Hong Kong, baseline TST reaction sizes in primary school children independently predicted subsequent TB risks (13.0, 18.8, 22.5, 280.4 per 100,000 person-years for reaction size of $0-4,5-9,10-14$, and $\geq 15 \mathrm{~mm}$, respectively), despite a $99 \%$ neonatal BCG vaccination coverage rate. ${ }^{50}$ For those with TST reaction size $\geq 15 \mathrm{~mm}$, the incidence of TB was significantly higher beyond the age of 15 years than before that age (608.1 vs. 37.5 per 100,000 person-year). The sharp increase in TB risk after the age of 15 coincided with the upsurge of TB often observed in late adolescence, possibly reflecting some ill-characterized age-dependent changes in host immunity.

Combating drug-resistant TB-An editorial article attributed the emergence of various forms of drug-resistant TB in India, a country with the highest number of TB cases, to the iatrogenic selection of progressively resistant strains through inadequate treatment in both public and private sectors. ${ }^{51}$ Although directly observed therapy-short course (DOTS) is used in the public sector, a "category 2" regimen, with the addition of only streptomycin to the standard four-drug short course ("category 1" regimen), is offered to patients who failed first-line treatment. Limited access to DOTS-Plus projects in the public sector forces the vast majority of MDR-TB patients to seek treatment from private practitioners, where 
unregulated use of second line drugs fuels the rapidly evolving epidemic of drug resistance, leading progressively to MDR-TB, extensively drug-resistant (XDR)-TB and ultimately totally drug-resistant (TDR)-TB.

Worry levels of bacillary resistance to the anti-tuberculous drugs have also been reported in China, where a national survey showed $5.7 \%$ of new culture-positive TB cases and $25.6 \%$ of previously treated cases to be MDR-TB. ${ }^{52}$ In China alone, there were as many as 110,000 incident MDR-TB cases and 8,200 incident XDR-TB cases in 2007. Patients with multiple previous treatments and having received their last treatment in a TB hospital had the highest risk of MDR-TB (adjusted odds ratio, 13.3). However, the majority of MDR- and XDR-TB resulted from primary transmission, indicating secondary propagation of resistant strains within this most populous country of the world.

Walter et al. reviewed how recent basic science developments might contribute to the public health response to the global crisis of drug-resistant TB. ${ }^{53}$ Following elucidation of the genetic basis of resistance, new automated molecular tools are transforming the diagnosis of MDR-TB. Molecular epidemiological approaches have also provided new insights into the transmission of the disease. The transmission of MDR- and XDR-TB was examined in Hong Kong using conventional household contact tracing and restriction fragment length polymorphism (RFLP) analysis. ${ }^{54}$ XDR-TB significantly increased household transmission of TB, as compared to other MDR-TB, likely reflecting the differing periods of infectivity. Of 20 retrievable isolates from 27 XDR-TB index cases, RFLP analysis showed clustering among $13(65.0 \%)$, with 11 (55\%) due to recent transmission by the "n-1" method. An identifiable household source was identified in only $3(27.2 \%)$ of the 11 recently transmitted cases, thus pointing to predominant transmission of this dangerous form of TB outside the household setting.

In a meta-analysis pooling individual data of 9,153 MDR-TB patients across 32 observational studies, later generation fluoroquinolones (adjusted odds ratio [aOR]: 2.5), ofloxacin (aOR: 2.5), ethionamide or prothionamide (aOR: 1.7), use of four or more likely effective drugs in the initial intensive phase (aOR: 2.3), and three or more likely effective drugs in the continuation phase (aOR: 2.7 ) were independently associated with treatment success. ${ }^{55}$ Notwithstanding, treatment options are often severely limited by resistance or intolerance to the above drugs. New drugs are therefore needed for effective treatment of highly resistant forms of TB. ${ }^{51}$ Linezolid has been found to be useful for achieving culture conversion among patients with XDR-TB. ${ }^{56}$ However, adverse effects were found in $82 \%$ of patients, even though fewer were observed with a linezolid dosage of $300 \mathrm{mg} / \mathrm{day}$ than 600 $\mathrm{mg} /$ day. Delamanid (OPC-67683), a nitro-dihydro-imidazooxazole derivative that inhibits mycolic acid synthesis, has shown potent in vitro and in vivo activity against drug-resistant strains of M. tuberculosis. In a randomized trial, $45.4 \%$ of MDR-TB patients who received $100 \mathrm{mg}$ of delamanid twice daily on top of a background drug regimen had sputum-culture conversion in liquid medium at 2 months, as compared with only $29.6 \%$ of similar patients who received placebo plus a background drug regimen. ${ }^{57}$ However, the observed 2-month culture conversion rate still fell short of what can be achieved by the standard regimen containing isoniazid, rifampicin and pyrazinamide for fully drug-sensitive disease. Further research on a combination of new TB drugs is therefore required to maximize treatment success and reduce the risk of acquired drug resistance. ${ }^{53}$ Promising 14-day early bactericidal activity has recently been reported for a combination of PA-824, moxifloxacin and pyrazinamide, making it a potential candidate in the ongoing search for a regimen suitable for treating both drug-sensitive TB and MDR-TB. ${ }^{58}$ 


\section{PLEURAL DISEASES}

\section{José M. Porcel}

Pleural fluid analysis-Pleural fluid analysis represents the most important step in the evaluation of the potential cause of pleural effusions. In a comprehensive review by Sahn, the clinical presentation, the natural course of specific pleural effusions, and the contribution of pleural fluid analyses to the definitive diagnosis are discussed. ${ }^{59}$ The time course of recurrence of pleural effusions following therapeutic thoracentesis may be of value to the clinician when diagnosis is doubtful. For instance, reaccumulation of pleural fluid in 24-72 hours of the procedure is typically seen in hepatic hydrothoraces, unexpandable lungs, peritoneal dialyses, and chylothoraces. Chronicity of pleural effusions also limits the differential diagnosis. There are only a few causes of persistent benign pleural effusions, including benign asbestos pleural effusions, trapped lung due to previous inflammatory conditions (eg., postcardiac surgery, rheumatoid pleuritis, uremic pleuritis, hemothorax, infectious effusions), and chylothoraces secondary to congenital or acquired lymphatic disorders (eg., yellow nail syndrome, lymphangioleiomyomatosis)..$^{59}$

An initial step in the evaluation of pleural effusions is to determine whether the fluid is a transudate or an exudate, for which Light's criteria are universally used. However, in one study $29 \%$ of 364 heart failure-related effusions and $18 \%$ of 102 hepatic hydrothoraces met Light's exudative criteria by a small margin. ${ }^{60} \mathrm{~A}$ serum to pleural fluid albumin gradient $>1.2 \mathrm{~g} / \mathrm{dL}$ correctly identified $83 \%$ of the mislabeled cardiac effusions, whereas a pleural fluid to serum albumin ratio $<0.6$ enabled the proper classification of $77 \%$ of the miscategorized cirrhosis-associated effusions. Notably, only $55 \%$ and $61 \%$ respectively of cardiac and hepatic false exudates would have been identified by a pleural fluid protein gradient $>3.1 \mathrm{~g} / \mathrm{dL}$. According to this study, the latter should no longer be recommended as the parameter of choice for mislabeled transudates.

Adenosine deaminase (ADA) is a well-established biomarker of pleural TB. However, more than $40 \%$ of parapneumonic effusions and $10 \%$ of non-tuberculous lymphocytic exudates exhibit pleural ADA activity higher than the cutoff set for TB (usually $35 \mathrm{U} / \mathrm{L}$ ) ${ }^{61}$ Even though more than $80 \%$ of parapneumonic effusions show a predominance of polymorphonuclear leukocytes and $90 \%$ of TB effusions are lymphocytic, ${ }^{62}$ there may be some overlapping. A pilot study found that in 44 effusions with pleural fluid ADA concentrations greater than $50 \mathrm{U} / \mathrm{L}$, pleural fluid potassium levels exceeded $5 \mathrm{mEq} / \mathrm{L}$ only in $1(5.5 \%)$ of 18 tuberculous against $15(57.5 \%)$ of 26 nontuberculous fluids. ${ }^{63}$ These latter 15 effusions were all parapneumonics. The study was small and retrospective, but because of the simplicity of potassium measurement as compared with the ADA isoenzymes, it merits future confirmation.

Parapneumonic effusions-Pleural infection is rising in incidence across all age groups worldwide. A population-based study in Taiwan analyzed the characteristics of 26,385 patients with pleural infections between 1997 and $2008 .{ }^{64}$ Over the 12-year period, there was an increase in the median age (from 60 to 65 years) and disease severity of the patients, incidence of pleural infections (from 5.2/100,000 in 1997 to 8.4-9.6/100,000 between 2002 and 2008), resource utilization (CT, chest catheters, intrapleural fibrinolytics, surgery), and hospital charges per patient, yet a decrease in 30-day mortality (from $15 \%$ to $13.1 \%$ ). ${ }^{64}$ Decline in case-fatality rate may be explained by advances in the therapeutic and supportive management of pleural infections.

Streptococcus pneumoniae is the leading cause of pediatric empyema and the second most common causative organism in adult empyema. The incidence of pneumococcal empyema in children and young adults has increased as the result of the emergence of serotypes 1 and 
3 , which are not included in the 7-valent pneumococcal conjugate vaccine. ${ }^{65}$ Therefore, it is essential to be aware of changes in serotype pneumococcal epidemiology, particularly in the setting of new vaccine developments. In this sense, the use of molecular techniques (PCR) for identifying pneumococcal serotypes in culture-negative childhood empyemas may potentially result in additional serotype information compared to conventional surveillance based on standard cultures alone. In a prospective study of 89 children with empyema, $S$. pneumoniae was isolated in $6 \%$ of the cases by pleural fluid cultures and in $54 \%$ by nucleic acid testing. ${ }^{66}$ Serotype identification in culture negative pleural fluids by a multiplex PCRbased reverse line blot assay demonstrated a disproportionate representation of serotypes 3 (31\%) and $1(14 \%)$ compared with the percentages identified from 331 invasive pneumococcal disease cases (mainly from blood) which had been sent to a national surveillance system ( $4 \%$ and $2.4 \%$ respectively) during the same time period. ${ }^{66}$

The identification of non-purulent-appearing parapneumonic effusions that require chest tube drainage (ie., complicated parapaneumonics) is a clinical challenge. Clinical guidelines advocate the use of pleural fluid $\mathrm{pH}$ (or glucose) and cultures as criteria for instituting tube thoracostomy. Nevertheless, about $40 \%$ and $80 \%$ of patients respectively, who are later treated by chest tube drainage do not have pleural fluid acidosis or positive cultures. ${ }^{67}$ Due to the low sensitivity of these classical parameters, new biomarkers of infection have been tested for discriminating purposes. Among them is the proinflammatory marker C-reactive protein (CRP). One study measured serum and pleural fluid CRP in 54 patients with nonpurulent parapneumonic effusions, of which 20 were complicated and 34 uncomplicated. ${ }^{68}$ Notably, the presence of both a serum CRP $>83 \mathrm{mg} / \mathrm{L}$ and a pleural fluid $\mathrm{pH}<7.20$ strongly argued for complicated effusions (likelihood ratio (LR) positive $=23.2$ ), whereas a patient in whom the serum CRP is $<83 \mathrm{mg} / \mathrm{L}$ or the pleural fluid $\mathrm{pH}$ is $>7.20$ will most likely have a non-complicated effusion (LR negative virtually 0 ). Eleven (20\%) patients developed residual pleural thickening $>1 \mathrm{~cm} 6$ months or more after discharge. This complication was independently predicted (LR positive of 6.6 ) by an initial serum CRP level $>150 \mathrm{mg} / \mathrm{L} .{ }^{68}$

Pleural procedures-Chest drain insertion is a procedure that may be required under a number of circumstances, such as pneumothorax, empyema, hemothorax and malignant effusions. Traditionally, risk of bleeding by an uncontrolled coagulopathy has been considered a relative contraindication for drainage. Clopidogrel is an antiplatelet agent used for the treatment of myocardial infarction and stroke and, in combination with aspirin, is prescribed in patients with acute coronary syndrome and coronary artery stents. Guidelines recommend discontinuing clopidogrel for 5-7 days prior to invasive procedures to allow new platelets to replace those that have undergone irreversible inhibition. ${ }^{69}$ A retrospective analysis of 24 patients who underwent small-bore chest tube ( $\triangle 6 \mathrm{~F}$ ) insertions under the guidance of ultrasound while receiving clopidogrel therapy documented no bleeding complications. ${ }^{70}$ Therefore, other than being impractical, withholding pleural drainage until the antiplatelet effect dissipates seems unnecessary.

Medical thoracoscopy or pleuroscopy is currently the gold standard for the diagnosis and treatment of many pleural conditions. According to the Thoracic Society of Australia and New Zealand (TSANZ) guidelines, the recommended minimum number of supervised procedures for training is 20 pleuroscopies, although this does not necessarily establish competency. ${ }^{71}$ A more complex and invasive procedure is VATS, which is performed by thoracic surgeons rather than interventional pulmonologists. VATS allows the performance of complex therapeutic pleural procedures, previously reserved to open thoracotomy. One illustrative example is the management of pleural metastases in patients with OC. ${ }^{72}$ The presence of malignant pleural effusions characterizes stage IV OC. Optimal tumor debulking (no residual tumor mass $>1 \mathrm{~cm}$ ) is the primary goal in patients with OC, since it is associated with increased survival. Surgical cytoreduction should ideally involve not only 
the abdominal structures, but also the tumor burden of the pleural space. Thus, VATS may have a significant impact on the global therapeutic strategy in patients with suspected or confirmed malignant pleural effusions before a planned abdominal exploration. It is proposed that a VATS examination be performed to quantify pleural tumor burden in good surgical candidates with moderate to large pleural effusions. When feasible, maximum removal of gross pleural nodules should be attempted, followed by abdominal surgery. If pleural disease is unresectable, pleurodesis, neoadjuvant chemotherapy and interval abdominal debulking is the logical choice. ${ }^{72}$

\section{BRONCHOSCOPIC INTERVENTION AND IMAGING}

\section{Pyng Lee}

Bronchoscopic intervention-Over the past year comprehensive reviews, guidelines, and interesting studies on thoracic imaging, diagnostic and interventional bronchoscopy procedures have been published. The first was an excellent appraisal of various bronchoscopic imaging modalities by Ohtani et $a I^{73}$ Following the invention of the fiberoptic bronchoscope by Shigeto Ikeda, bronchoscopy has revolutionized the practice of pulmonary medicine. White light bronchoscopy uses the reflectance and scattering properties of light from tissue to define the macroscopic appearance of airways. Autofluorescence bronchoscopy depends on the concentration of fluorophores in the bronchial tissue, while confocal endomicroscopy allows cellular imaging and optical coherence tomography three-dimensional imaging of the airways. Laser Raman spectroscopy defines not only microscopic structures, but also biochemical and molecular compositions of tissues in disease states.

In the largest postal survey conducted by the JRS to determine the use and complications associated with respiratory endoscopy, $90 \%$ responded. ${ }^{74}$ Total number of diagnostic flexible bronchoscopy procedures performed was 103,978 , mortality was $0.004 \%$, and complication rates between 0.51 and $2.06 \%$. The highest complication rate was observed in patients undergoing bronchoscopy for diffuse lung disease. Therapeutic bronchoscopy was performed in 3,020 patients, mainly for foreign body removal. Mortality from therapeutic bronchoscopy was $0.03 \%$ where 1 patient died of hemorrhage from an expandable metallic stent insertion. In 3,689 patients who underwent EBUS-TBNA of the hilar and/or mediastinal lesions, complications occurred in $17(0.46 \%)$, of which pulmonary infections were the most common. Transbronchial lung biopsy of solitary pulmonary lesions with radial EBUS was performed in 3,853 cases, hemorrhage occurring in $0.73 \%$ followed by pneumothorax in $0.63 \%$. Flex-rigid pleuroscope was used in 1,563 patients, and bleeding was reported in $1 \%$ of those who underwent biopsy without electrocautery. Half of facilities reported breakages of bronchoscopes. The authors concluded that respiratory endoscopy was safe overall. However, operators and nurses should be trained to handle complications that may arise from new techniques. ${ }^{74}$

The TSANZ developed guidelines for training in advanced interventional procedures, since published literature shows conflicting results about the minimum number of cases to assure competency and proficiency. Basic bronchoscopy skills, reading, simulated training and testing as well as the audit of procedural outcome and complications were established to achieve a rounded approach. In addition, the guidelines recommend that advanced interventional procedures should be pursued only if there is a realistic expectation that the trainee will achieve sufficient proficiency to perform them without supervision. The guidelines also provide direction for maintaining the skill set thereafter, as brief exposures to these procedures during pulmonary and critical care fellowship programs or training courses are inadequate to achieve competency. ${ }^{75}$ 
Stather et al. showed that training with an EBUS-TBNA simulator led to the rapid acquisition of clinical skills comparable to conventional practice on patients, differing only in the time needed to intubate. ${ }^{76}$ Skills learned with the simulator were transferable, and could impact clinical technique performance. Simulators can complement training and minimize burden of procedural learning on patients.

EBUS has an established role in cancer staging. Aspirates obtained with EBUS-TBNA are sufficient for molecular markers to allow personalized therapy. In one study, EBUS-TBNA samples were not only adequate to diagnose metastatic melanoma in the hilar and mediastinal LNs, but also for mutations of the BRAF gene. ${ }^{77}$

Unlike EBUS-TBNA, conventional TBNA is operator dependent and has variable yield. In one study, no difference in the diagnostic accuracy was found between liquid-based cytology and conventional smear cytology. ${ }^{78}$ TBNA had an overall diagnostic sensitivity of $63.6 \%$ (56/88), and higher yields were achieved for small cell lung cancers than for NSCLCs, subcarinal nodal stations, and LNs measuring $>2 \mathrm{~cm}$ on CT. ${ }^{78}$

Imaging - CT pulmonary angiography (CTPA) is the gold standard for assessing the extent of pulmonary artery obstruction in patients with chronic thromboembolic pulmonary hypertension. One study compared SPECT perfusion scanning and CTPA before pulmonary endarterectomy in 12 patients. Anatomical perfusion defects observed with SPECT were matched against pulmonary arterial filling defects by CTPA, and correlated with pathology. Clinicopathological correlation showed 140 obstructed and 40 unobstructed lung segments. SPECT identified $62 \%$ of obstructed and $72 \%$ of unobstructed segments, while CTPA detected $47.8 \%$ of obstructed and $80 \%$ of unobstructed segments. Sensitivity was higher for SPECT than CTPA. ${ }^{79}$

Kurashima et al. investigated whether airway caliber and wall thickness as determined by CT correlated to pulmonary function in 63 patients with asthma, 46 COPD, 23 asthma with emphysema, and 61 normal controls. Curved multiplanar reconstruction software was applied to measure airway dimensions from the $3^{\text {rd }}$ to $6^{\text {th }}$ generation bronchi of the right lower posterior bronchus. Patients with COPD had increased wall thickness, but no airway narrowing from the $3^{\text {rd }}$ to $6^{\text {th }}$ generation bronchi. On the other hand, patients with asthma or asthma with emphysema demonstrated smaller bronchial inner diameters of the $3^{\text {rd }}$ to $6^{\text {th }}$ generation bronchi. Airway luminal area of the $5^{\text {th }}$ and $6^{\text {th }}$ generation bronchi correlated with pulmonary function in patients with stable asthma and asthma with emphysema, respectively. ${ }^{80}$

In one study, 25 asthmatics and 8 healthy volunteers underwent spirometry, plethysmography and hyperpolarized ${ }^{3} \mathrm{He}$ magnetic resonance imaging (MRI) before and after a methacholine challenge test and $25 \mathrm{~min}$ after salbutamol administration.

Heterogeneity in ventilation and ${ }^{3} \mathrm{He}$ apparent diffusion coefficients consistent with bronchoconstriction and gas trapping was demonstrated post-methacholine challenge test and resolved after salbutamol administration. ${ }^{3} \mathrm{He}$ MRI may emerge as a dynamic test for asthma where spirometry measurements are unyielding. ${ }^{81}$

Until recently, the imaging modality of choice for clinical diagnosis and monitoring of respiratory diseases has been high-resolution CT (HRCT). The MRI does not surpass HRCT in speed, image contrast and content or spatial resolution. Proton MRI depends on proton density of tissues. Lung has low tissue density and is devoid of water. Thus, thoracic MRI, even when optimized for lung results, is devoid of tissue and morphological information. Moreover, lung consists of millions of air-tissue interfaces for gas exchange that give rise to so-called magnetic 'susceptibility artifacts'. For these reasons, pulmonary MRI may never 
replace HRCT for lung disease diagnoses. The lung, on the other hand, is the most radiosensitive organ in the chest and serial HRCTs increase the risk of cumulative radiation, especially in children. Chest MRI was compared with HRCT in the evaluation of children with non-cystic fibrosis chronic lung diseases. Both techniques were found to be equivalent for the identification of bronchiectasis, mucus plugging, peribronchial wall thickening, consolidation, bullae, abscesses and emphysema. Owing to its relatively low radiation risk, MRI could represent an alternative imaging modality. ${ }^{82}$

Spirometry and body plethismography represent current clinical standards for assessing and monitoring disease severity in pulmonary diseases. However, they do not explain variable clinical manifestations or the relationship between lung structure and function. Washko et al. provide details on the imaging modalities being used for the non- invasive study of lung anatomy and function, focusing on CT and MRI. ${ }^{83}$

\section{Acknowledgments}

MIR's time is partially protected by Award Number K23HL096054 from the National Heart, Lung, and Blood Institute. The content of MIR's contribution on respiratory infections is solely the responsibility of the author and does not necessarily represent the official views of the National Heart, Lung, And Blood Institute, the National Institutes of Health, or the Department of Veterans Affairs.

\section{References}

1. Jemal A, Bray F, Center MM, et al. Global cancer statistics. CA Cancer J Clin. 2011; 61:69-90. [PubMed: 21296855]

2. Lim WY, Seow A. Biomass fuels and lung cancer. Respirology. 2012; 17:20-31. [PubMed: 22008241]

3. Lissowska J, Bardin-Mikolajczak A, Fletcher T, et al. Lung cancer and indoor pollution from heating and cooking with solid fuels: the IARC international multicentre case-control study in Eastern/Central Europe and the United Kingdom. Am J Epidemiol. 2005; 162:326-33. [PubMed: 16014775]

4. Hosgood HD 3rd, Boffetta P, Greenland S, et al. In-home coal and wood use and lung cancer risk: a pooled analysis of the International Lung Cancer Consortium. Environ Health Perspect. 2010; 118:1743-7. [PubMed: 20846923]

5. Bae EY, Lee SY, Kang BK, et al. Replication of results of genome-wide association studies on lung cancer susceptibility loci in a Korean population. Respirology. 2012; 17:699-706. [PubMed: 22404340]

6. Aberle DR, Adams AM, Berg CD, et al. Reduced lung-cancer mortality with low-dose computed tomographic screening. N Engl J Med. 2011; 365:395-409. [PubMed: 21714641]

7. Spiro SG, Navani N. Screening for lung cancer: is this the way forward? Respirology. 2012; 17:237-46. [PubMed: 22142440]

8. Infante M, Lutman FR, Cavuto S, et al. Lung cancer screening with spiral CT: baseline results of the randomized DANTE trial. Lung Cancer. 2008; 59:355-63. [PubMed: 17936405]

9. Blanchon T, Bréchot JM, Grenier PA, et al. Baseline results of the Depiscan study: a French randomized pilot trial of lung cancer screening comparing low dose CT scan (LDCT) and chest Xray (CXR). Lung Cancer. 2007; 58:50-8. [PubMed: 17624475]

10. Schmid-Bindert G, Jiang H, Kähler G, Saur J, et al. Predicting malignancy in mediastinal lymph nodes by endobronchial ultrasound: A new ultrasound scoring system. Respirology. 2012; 17:1190-8. [PubMed: 22789110]

11. Marshall HM, Leong SC, Bowman RV, et al. The science behind the 7th edition Tumour, Node, Metastasis staging system for lung cancer. Respirology. 2012; 17:247-60. [PubMed: 21992710]

12. Travis WD, Brambilla E, Noguchi M, et al. International association for the study of lung cancer/ American thoracic society/European respiratory society international multidisciplinary classification of lung adenocarcinoma. J Thorac Oncol. 2011; 6:244-85. [PubMed: 21252716] 
13. Kadara H, Kabbout M, Wistuba II. Pulmonary adenocarcinoma: a renewed entity in 2011. Respirology. 2012; 17:50-65. [PubMed: 22040022]

14. Yanagisawa S, Morikawa N, Kimura Y, et al. Large-cell neuroendocrine carcinoma with epidermal growth factor receptor mutation: Possible transformation of lung adenocarcinoma. Respirology. 2012; 17:1275-7. [PubMed: 22943430]

15. Chen HY, Yu SL, Li KC, et al. Biomarkers and transcriptome profiling of lung cancer. Respirology. 2012; 17:620-6. [PubMed: 22372638]

16. Fujii T, Toyooka S, Ichimura K, et al. ERCC1 protein expression predicts the response of cisplatinbased neoadjuvant chemotherapy in non-small-cell lung cancer. Lung Cancer. 2008; 59:377-84. [PubMed: 17905465]

17. Tseden-Ish M, Choi YD, Cho HJ, et al. Disease-free survival of patients after surgical resection of non-small cell lung carcinoma and correlation with excision repair cross-complementation group 1 expression and genotype. Respirology. 2012; 17:127-33. [PubMed: 21899657]

18. Acloque H, Adams MS, Fishwick K, et al. Epithelial-mesenchymal transitions: the importance of changing cell state in development and disease. J Clin Invest. 2009; 119:1438-49. [PubMed: 19487820]

19. Thiery JP, Acloque H, Huang RY, et al. Epithelial-mesenchymal transitions in development and disease. Cell. 2009; 139:871-90. [PubMed: 19945376]

20. Sato M, Shames DS, Hasegawa Y. Emerging evidence of epithelial-to-mesenchymal transition in lung carcinogenesis. Respirology. 2012; 17:1048-59. [PubMed: 22452538]

21. Toyooka S, Toyooka KO, Maruyama R, et al. DNA methylation profiles of lung tumors. Mol Cancer Ther. 2001; 1:61-7. [PubMed: 12467239]

22. Kase S, Sugio K, Yamazaki K, et al. Expression of E-cadherin and beta-catenin in human nonsmall cell lung cancer and the clinical significance. Clin Cancer Res. 2000; 6:4789-96. [PubMed: 11156236]

23. Giroux Leprieur E, Lavole A, Ruppert AM, et al. Factors associated with long-term survival of patients with advanced non-small cell lung cancer. Respirology. 2012; 17:134-42. [PubMed: 21943088]

24. Van TT, Hanibuchi M, Goto H, et al. SU6668, a multiple tyrosine kinase inhibitor, inhibits progression of human malignant pleural mesothelioma in an orthotopic model. Respirology. 2012; 17:984-90. [PubMed: 22574723]

25. Hsu AC, See HV, Hansbro PM, et al. Innate immunity to influenza in chronic airways diseases. Respirology. 2012; 17:1166-75. [PubMed: 22616906]

26. Miyashita N, Kawai Y, Akaike H, et al. Influence of age on the clinical differentiation of atypical pneumonia in adults. Respirology. 2012; 17:1073-9. [PubMed: 22563979]

27. Yanagihara K, Kohno S, Matsusima T. Japanese guidelines for the management of communityacquired pneumonia. Int J Antimicrob Agents. 2001; 18:S45-8. [PubMed: 11574195]

28. Yin YD, Zhao F, Ren LL, et al. Evaluation of the Japanese Respiratory Society guidelines for the identification of Mycoplasma pneumoniae pneumonia. Respirology. 2012; 17:1131-6. [PubMed: 22805282]

29. Abisheganaden J, Ding YY, Chong WF, et al. Predicting mortality among older adults hospitalized for community-acquired pneumonia: An enhanced Confusion, Urea, Respiratory rate and Blood pressure score compared with Pneumonia Severity Index. Respirology. 2012; 17:969-75. [PubMed: 22574694]

30. Kasamatsu Y, Yamaguchi T, Kawaguchi T, et al. Usefulness of a semi-quantitative procalcitonin test and the A-DROP Japanese prognostic scale for predicting mortality among adults hospitalized with community -acquired pneumonia. Respirology. 2012; 17:330-6. [PubMed: 22059525]

31. Brown SM, Jones JP, Aronsky D, et al. Relationships among initial hospital triage, disease progression and mortality in community-acquired pneumonia. Respirology. 2012; 17:1207-13. [PubMed: 22805170]

32. Mandell LA, Wunderink RG, Anzueto A, et al. Infectious Diseases Society of America; American Thoracic Society. Infectious Diseases Society of America/American Thoracic Society consensus guidelines on the management of community-acquired pneumonia in adults. Clin Infect Dis. 2007; 1:S27-72. [PubMed: 17278083] 
33. Woodhead M, Blasi F, Ewig S, et al. Joint Taskforce of the European Respiratory Society and European Society for Clinical Microbiology and Infectious Diseases. Guidelines for the management of adult lower respiratory tract infections. Clin Microbiol Infect. 2011; 17:E1-59. [PubMed: 21951385]

34. Yoon YK, Kim EJ, Chun BC, et al. Prescription of antibiotics for adults hospitalized with community-acquired pneumonia in Korea in 2004: a population-based descriptive study. Respirology. 2012; 17:172-9. [PubMed: 21995414]

35. Llor C, Bayona C, Hernandez S, et al. Comparison of adherence between twice- and thrice-daily regimens of oral amoxicillin/clavulanic acid. Respirology. 2012; 17:687-92. [PubMed: 22390206]

36. Hodge S, Reynolds PN. Low-dose azithromycin improves phagocytosis of bacteria by both alveolar and monocyte-derived macrophagesin chronic obstructive pulmonary disease subjects. Respirology. 2012; 17:802-7. [PubMed: 22288725]

37. Albert RK, Connett J, Bailey WC, et al. Azithromycin for prevention of exacerbations of COPD. N Engl J Med. 2011; 365:689-98. [PubMed: 21864166]

38. Wenzel RP, Fowler AA 3rd, Edmond MB. Antibiotic prevention of acute exacerbations of COPD. N Engl J Med. 2012; 367:340-7. [PubMed: 22830464]

39. Hansbro PM, Jarnicki AG. Macrolides for macrophages in chronic obstructive pulmonary disease. Respirology. 2012; 17:739-40. [PubMed: 22563902]

40. Ding FM, Zhu SL, Shen C, et al. Low-dose clarithromycin therapy modulates CD4(+) T-cell responses in a mouse model of chronic Pseudomonas aeruginosa lung infection. Respirology. 2012; 17:727-34. [PubMed: 22404374]

41. Kawai Y, Miyashita N, Yamaguchi T, et al. Clinical efficacy of macrolide antibiotics against genetically determined macrolide-resistant Mycoplasma pneumoniae pneumonia in paediatric patients. Respirology. 2012; 17:354-62. [PubMed: 22077195]

42. Li H, Zhu S, He S, et al. Anti-inflammatory effects of moxifloxacin on rat airway smooth muscle cells exposed to allergen: Inhibition of extracellular-signal-regulated kinase and nuclear factorkappaB activation and of interleukin-8 and eotaxin synthesis. Respirology. 2012; 17:997-1005. [PubMed: 22616553]

43. Smith JA, Kauffman CA. Pulmonary fungal infections. Respirology. 2012; 17:913-26. [PubMed: 22335254]

44. Hardak E, Neuberger A, Yigla M, et al. Outcome of Pneumocystis jirovecii pneumonia diagnosed by polymerase chain reaction in patients without human immunodeficiency virus infection. Respirology. 2012; 17:681-6. [PubMed: 22390188]

45. Hojo M, Iikura M, Hirano S, et al. Increased risk of nontuberculous mycobacterial infection in asthmatic patients using long-term inhaled corticosteroid therapy. Respirology. 2012; 17:185-90. [PubMed: 21995339]

46. Koo HK, Lee JS, Jeong YJ, et al. Vitamin D deficiency and changes in serum vitamin D levels with treatment among tuberculosis patients in South Korea. Respirology. 2012; 17:808-13. [PubMed: 22449254]

47. Sun Q, Zhang Q, Xiao H, et al. Significance of the frequency of CD4+CD25+CD127- T-cells in patients with pulmonary tuberculosis and diabetes mellitus. Respirology. 2012; 17:876-82. [PubMed: 22524702]

48. Miao R, Li J, Sun Z, et al. Association between the CD209 promoter -336A/G polymorphism and susceptibility to tuberculosis: a meta-analysis. Respirology. 2012; 17:847-53. [PubMed: 22553928]

49. Jo KW, Jeon K, Kang YA, et al. Poor correlation between tuberculin skin tests and interferon- $\gamma$ assays in close contacts of patients with multidrug-resistant tuberculosis. Respirology. 2012; 17:1125-30. [PubMed: 22758779]

50. Leung CC, Yew WW, Au KF, et al. A strong tuberculin reaction in primary school children predicts tuberculosis in adolescence. Pediatr Infect Dis J. 2012; 31:150-3. [PubMed: 21983215]

51. Udwadia ZF. Totally Drug Resistant Tuberculosis in India: who let the djinn out? Respirology. 2012; 17:741-2. [PubMed: 22564108]

52. Zhao Y, Xu S, Wang L, et al. National survey of drug-resistant tuberculosis in China. N Engl J Med. 2012; 366:2161-70. [PubMed: 22670902] 
53. Walter ND, Strong M, Belknap R, et al. Translating basic science insight into public health action for multidrug- and extensively drug-resistant tuberculosis. Respirology. 2012; 17:772-91. [PubMed: 22458269]

54. Leung EC, Leung CC, Kam KM, et al. Transmission of multidrug-resistant and extensively drugresistant tuberculosis in a metropolitan city. Eur Respir J. (in press).

55. Ahuja SD, Ashkin D, Avendano M, et al. Collaborative Group for Meta-Analysis of Individual Patient Data in MDR-TB. Multidrug Resistant Pulmonary Tuberculosis Treatment Regimens and Patient Outcomes: An Individual Patient Data Meta-analysis of 9,153 Patients. PLoS Med. 2012; 9:e1001300. [PubMed: 22952439]

56. Lee M, Lee J, Carroll MW, et al. Linezolid for treatment of chronic extensively drug-resistant tuberculosis. N Engl J Med. 2012; 367:1508-18. [PubMed: 23075177]

57. Gler MT, Skripconoka V, Sanchez-Garavito E, et al. Delamanid for multidrug-resistant pulmonary tuberculosis. N Engl J Med. 2012; 366:2151-60. [PubMed: 22670901]

58. Diacon AH, Dawson R, von Groote-Bidlingmaier F, et al. 14-day bactericidal activity of PA-824, bedaquiline, pyrazinamide, and moxifloxacin combinations: a randomised trial. Lancet. 2012; 380:986-93. [PubMed: 22828481]

59. Sahn SA. Getting the most from pleural fluid analysis. Respirology. 2012; 17:270-7. [PubMed: 22059482]

60. Bielsa S, Porcel JM, Castellote J, et al. Solving the Light's criteria misclassification rate of cardiac and hepatic transudates. Respirology. 2012; 17:721-6. [PubMed: 22372660]

61. Porcel JM, Esquerda A, Bielsa S. Diagnostic performance of adenosine deaminase activity in pleural fluid: a single-center experience with over 2100 consecutive patients. Eur J Intern Med. 2010; 21:419-23. [PubMed: 20816597]

62. Porcel JM. Pearls and myths in pleural fluid analysis. Respirology. 2011; 16:44-52. [PubMed: 20573057]

63. Kashiwabara K, Okamoto T, Yamane H. When pleural potassium exceeds $5.0 \mathrm{mEq} / \mathrm{L}$, high pleural adenosine deaminase levels do not necessarily indicate tuberculous pleuritis. Respirology. 2012; 17:92-8. [PubMed: 21883680]

64. Shen HN, Lu CH, Li CY. Epidemiology of pleural infections in Taiwan from 1997 through 2008. Respirology. 2012; 17:1086-93. [PubMed: 22757969]

65. Burgos J, Luján M, Falcó V, et al. The spectrum of pneumococcal empyema in adults in the early $21^{\text {st }}$ century. Clin Infect Dis. 2011; 53:254-61. [PubMed: 21765073]

66. Strachan RE, Cornelius A, Gilbert GL, et al. Pleural fluid nucleic acid testing enhances pneumococcal surveillance in children. Respirology. 2012; 17:114-9. [PubMed: 21848709]

67. Porcel JM, Bielsa S, Esquerda A, et al. Pleural fluid C-reactive protein contributes to the diagnosis and assessment of severity of parapneumonic effusions. Eur J Intern Med. 2012; 23:447-50. [PubMed: 22726374]

68. Skouras V, Boultadakis E, Nikoulis D, et al. Prognostic value of C-reactive protein in parapneumonic effusions. Respirology. 2012; 17:308-14. [PubMed: 21995428]

69. Patel IJ, Davidson JC, Nikolic B, et al. Consensus guidelines for periprocedural management of coagulation status and hemostasis risk in percutaneous image-guided interventions. J Vasc Interv Radiol. 2012; 23:727-36. [PubMed: 22513394]

70. Abouzgheib W, Shweihat YR, Meena N, et al. Is chest tube insertion with ultrasound guidance safe in patients using clopidogrel? Respirology. 2012; 17:1222-4. [PubMed: 22817765]

71. Fielding D, Phillips M, Robinson P, et al. Advances interventional pulmonology procedures: training guidelines form the Thoracic Society of Australia and New Zealand. Respirology. 2012; 17:1176-89. [PubMed: 22897225]

72. Porcel JM, Díaz JP, Chi DS. Clinical implications of pleural effusions in ovarian cancer. Respirology. 2012; 17:1060-7. [PubMed: 22458298]

73. Ohtani K, Lee A, Lam S. Frontiers in bronchoscopic imaging. Respirology. 2012; 17:261-9. [PubMed: 22126413]

74. Asano F, Aoe M, Ohsaki Y, et al. Deaths and complications associated with respiratory endoscopy: a survey by the Japan Society for Respiratory Endoscopy in 2010. Respirology. 2012; 17:478-85. [PubMed: 22222022] 
75. Fielding D, Philips M, Robinson P, et al. Advanced interventional pulmonary procedures: training guidelines from the Thoracic Society of Australia and New Zealand. Respirology. 2012; 17:117689. [PubMed: 22897225]

76. Stather DR, MacEachern P, Chee A, et al. Evaluation of clinical endobronchial ultrasound skills following clinical versus simulation training. Respirology. 2012; 17:291-9. [PubMed: 21943051]

77. Jennings BR, Milward MJ, Amanuel B, et al. Role of endobronchial ultrasound in diagnosis and molecular assessment of metastatic melanoma. Respirology. 2012; 17:991-6. [PubMed: 22617000]

78. Hou G, Yin Y, Wang W, et al. Clinical impact of liquid-based cytology test on diagnostic yields from transbronchial needle aspiration. Respirology. 2012; 17:1225-8. [PubMed: 22882625]

79. Soler X, Kerr KM, Marsh JJ, Renner JW, et al. Pilot study comparing SPECT perfusion scintigraphy with CT pulmonary angiography in chronic thromboembolic pulmonary hypertension. Respirology. 2012; 17:180-4. [PubMed: 21899658]

80. Kurashima K, Hoshi T, Takayanagi N, et al. Airway dimensions and pulmonary function in chronic obstructive pulmonary disease and bronchial asthma. Respirology. 2012; 17:79-86. [PubMed: 21883679]

81. Costella S, Kirby M, Maksym G, et al. Regional pulmonary response to a methacholine challenge using hyperpolarized ${ }^{3} \mathrm{He}$ magnetic resonance imaging. Respirology. 2012; 17:1237-46. [PubMed: 22889229]

82. Montella S, Maglione M, Bruzzese D, et al. Magnetic resonance imaging is an accurate and reliable method to evaluate non-cystic fibrosis paediatric lung disease. Respirology. 2012; 17:8791. [PubMed: 21943039]

83. Washko GR, Parraga G, Coxson HO. Quantitative pulmonary imaging using computed tomography and magnetic resonance imaging. Respirology. 2012; 17:432-44. [PubMed: 22142490] 